\title{
Mediastinal lymph node staging by EBUS-TBNA and CT: Characteristics of lymph node enlargement caused by anthracosis and other benign conditions
}

\author{
J ohannes Kirchner ${ }^{1}$, Michael Broll ${ }^{2}$, Philipp Mueller ${ }^{1}$, Christoph Murach ${ }^{2}$, Sven Stückradt ${ }^{1}$, \\ Natalia Pomjanski ${ }^{3}$, Stepfan Biesterfeld ${ }^{3}$, Dieter Liermann ${ }^{4}$, Ralph Kickuth ${ }^{5}$
}

1. Department of Diagnostic and Interventional Radiology, Allgemeines Krankenhaus Hagen, Germany. 2. Department of Medicine, Klinikum Wedau Duisburg, Germany. 3. Department of Cytopathology, Heinrich Heine Universität Düsseldorf, Germany. 4. Department of Diagnostic and Interventional Radiology and Nuclearmedicine, Katholisches Marienhospital Herne Universitätsklinikum der Ruhr-Universität Bochum, Germany. 5. Department of Radiology, Universitätsklinikum Würzburg, Germany.

Correspondence: Johannes Kirchner, Professor. Address: Department of Diagnostic and Interventional Radiology. Allgemeines Krankenhaus Hagen Grünstraße 35 D 58095 Hagen, Germany. Email: Kirchner@akh-hagen.de

Received: January 23, 2014

DOI : $10.5430 /$ ijdi.v1n2p64
Accepted: April 7, 2014

URL: http://dx.doi.org/10.5430/ijdi.v1n2p64

\section{Abstract}

Objectives: Aim of this comparative study was to assess the accuracy of computed tomography (CT) and endobronchial ultrasound guided transbronchial needle aspiration (EBUS-TBNA) for mediastinal lymph node staging in cases of lymph node enlargement due to anthracosis and other benign conditions.

Methods: In a retrospective analysis we report on the MSCT findings of 39 patients (28 males, 11 females) with EBUS-TBNA confirmed diagnosis of 53 enlarged lymph nodes due to anthracosis. A control group comprised 20 consecutive patients with 27 enlarged lymph nodes (11 males, 9 females) due to chronic lymphadenopathy $(\mathrm{n}=14)$ or sarcoidosis $(n=13)$.

Results: No significant differences were observed between the two groups regarding size (mean short axis diameter $13.7 \mathrm{~mm}$ vs. $14.5 \mathrm{~mm}$ ), shape (most often oval) or presence of lymph node confluence (32.1\% vs. $33.3 \%$ ), contrast enhancement (3.8\% vs. 3.7\%), and fatty involution (3.8\% vs. 3.7\%). In comparison with the control group anthracotic lymph nodes were significantly less often ill-defined in EBUS (5.7 vs. 25.9, $p=0.025)$ as well as in CT (1.9\% vs. 18.5\%, $p=0.01)$, but more often showed calcifications in CT ( $24.5 \%$ vs. $3.7 \%, p=0.017)$. Lymph node colliquation was seen neither in anthracosis nor in other benign conditions.

Conclusions: Mediastinal lymph node enlargement due to anthracosis, lymphadenopathy and sarcoidosis show some different findings in EBUS and CT but cannot definitely be differentiated.

Advances in knowledge: Radiologists should be aware of mediastinal lymph node enlargement due to anthracosis.

\section{Keywords}

EBUS-TBNA, Computed tomography, Mediastinum, Lymph node, Anthracosis, Sarcoidosis, Lymphadenopathy 


\section{I ntroduction}

Although the CT finding of enlarged mediastinal lymph nodes in cases of lung cancer is commonly interpreted as an indicator for metastatic disease, it is also seen in benign conditions like sarcoidosis, chronic heart failure, COPD and pneumoconiosis ${ }^{[1-8]}$. While the occurrence of enlarged calcified mediastinal lymph nodes has been generally accepted as a sign of pneumoconiosis in coal workers ${ }^{[6]}$, to date - perhaps as a result of the decline of the coal mining and steel industries in the western countries - less attention has been paid to the impact of dust on mediastinal lymph node staging in cancer patients. Since anthracosis is a form of pneumoconiosis not only caused by occupational exposition to coal dust, but also by cigarette smoking and environmental pollution ${ }^{[9]}$ radiologists should be aware of mediastinal lymph node enlargement also in these conditions.

Aim of this comparative study was to demonstrate the findings of EBUS-TBNA and chest CT in benigne enlarged mediastinal lymph nodes due to anthracosis, lymphadenopathy and sarcoidosis and to assess the impact of both methods for mediastinal lymph node staging in cases of lymph node enlargement due to these benign conditions.

\section{Material and methods}

We performed a retrospective study on all consecutive patients of our institutions with the EBUS-TBNA confirmed cytological diagnosis of lymph node anthracosis, chronic lymphadenitis or sarcoidosis between April 2009 and April 2012.

Multidetector CT examinations (MSCT) were carried out using a Siemens Somatom 64 (Siemens Medical Solutions, Forchheim, Germany) or a Toshiba Aquilion 64 (Toshiba Medical Systems, Tokyo, Japan) CT system. Images were obtained at full inspiration using a $64 \mathrm{~mm} \times 0.75 \mathrm{~mm}$ slice collimation with a tube voltage of $120 \mathrm{kV}$. The tube current $(\mathrm{mA})$ was adjusted in relation to patient attenuation by means of the Care Dose ${ }^{\circledR}$ modus (Siemens Medical Solutions) or the Sure Exposure ${ }^{\circledR}$ modus (Toshiba Medical Systems). The reconstruction slice thickness was 3-5mm. During MDCT 100$120 \mathrm{ml}$ of $300 \mathrm{mg}$ or $400 \mathrm{mgI} / \mathrm{l}$ contrast medium were generally administered IV at a rate of $2-3 \mathrm{ml} / \mathrm{s}$ with a power injector followed by a normal saline "chaser".

Retrospective evaluation of computed tomographic examinations was performed consensually by two board certified radiologists (JK, RK). Mediastinal and hilar lymph node evaluation comprised measurement of size (short axis and long axis). In this setting, lymph node enlargement was defined as showing a size of more than 7-11mm on the short axis depending on the regional nodal station following Glazer et al. ${ }^{[10]}$. Enlarged lymph nodes were assessed for density (prevalence of calcification or central low density as a sign of fatty involution), shape (oval, round) and contour (sharp, ill-defined). The presence of lymph node necrosis and noticeable contrast enhancement was documented. Nodal necrosis was considered present when an enlarged lymph node showed ill-defined low attenuation areas ${ }^{[11]}$. Noticeable lymph node enhancement was determined as nodal density $>60 \mathrm{HU}$ in CT. The site of the enlarged lymph nodes was documented on a standardized protocol according to the regional lymph node classification of the American Thoracic Society ${ }^{[12]}$. The evaluation comprised a correlation of CT findings and EBUS-reports. In this context, a determination which lymph nodes in CT had been aspirated by TBNA had been performed.

\subsection{EBUS-TBNA}

Endobronchial ultrasound guided transbronchial needle aspiration (EBUS-TBNA) was performed with the patient under sedation or under general anesthesia as previously described ${ }^{[13]}$. A linear array $7.5 \mathrm{MHz}$ ultrasonic bronchoscope (CP EBUS, Olympus Medical Systems, Tokyo, Japan) with a $90^{\circ}$ angle of view was introduced orally or through an intubation tube. Firstly, suspicious lymph nodes, which had been previously documented in the computed tomographic examination, were localized by means of the endobronchial ultrasound of the regarding ATS region (see Figure 1). As suggested by Fujiwara et al ${ }^{[14]}$ lymph nodes were categorized according to the following characteristics: enlargement $(>1 \mathrm{~cm} /<1 \mathrm{~cm})$, 
shape (oval/ round), margin (distinct/indistinct), echogenicity (homogenous, heterogeneous) and presence or absence of central necrosis. After identification of the suspicious node transbronchial needle aspiration was performed using a 22G needle (model NA 201 SX 402, Olympus Medical Systems, Tokyo, Japan) under real time ultrasound guidance (see Figure 2). The aspirated material was prepared on glass slides, air dried and stained.

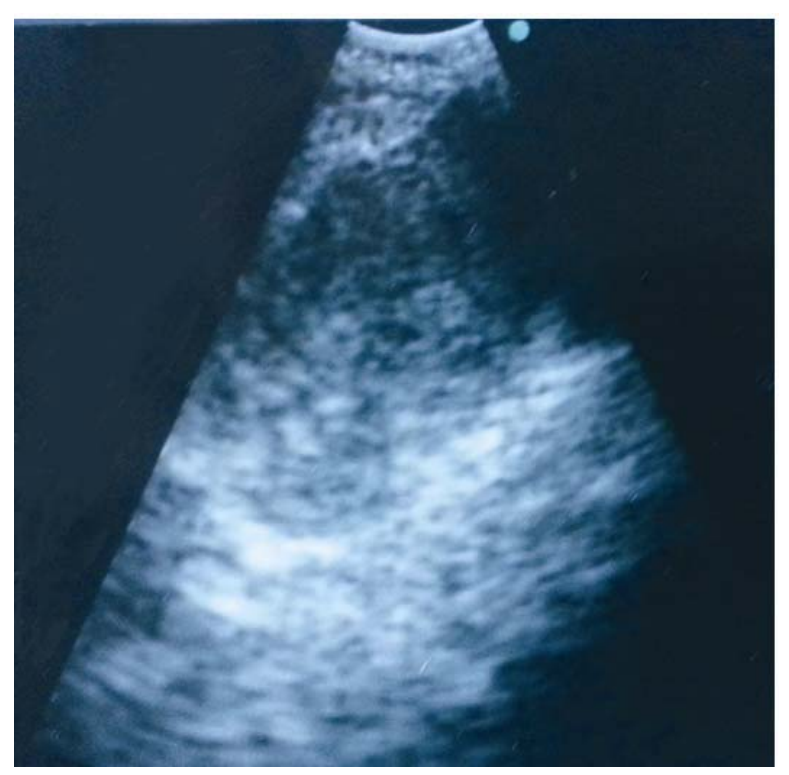

Figure 1. EBUS in an enlarged infracarinal lymph node (ATS region 7) due to anthracosis. The node shows a less or more oval shape, a homogenous echogenicity and clear defined margins.

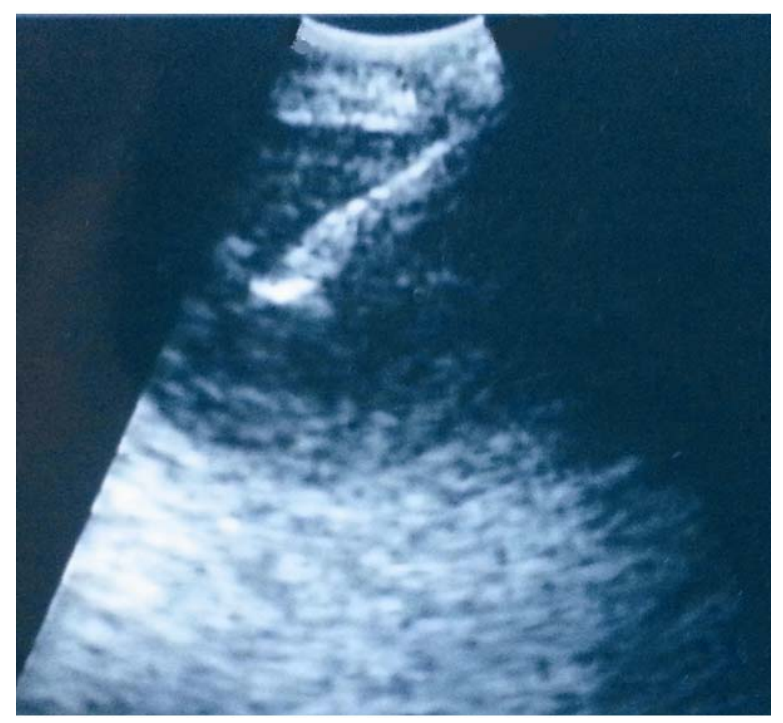

Figure 2. EBUS-TBNA in an enlarged paratracheal lymph node (ATS region 4R) due to anthracosis: oval, homogenous hypoechoic, good definable lymph node with the needle clearly visible in the center of the node.

We retrospectively evaluated the patients` medical reports with special regard to the underlying illnesses (diagnosis at admission and discharge), the presence of cigarette smoking or occupational dust exposure as well as definitive pathologic findings. 


\subsection{Statistical analysis}

Descriptive data were presented as means with ranges, if appropriate; categorical data were given as counts and percentages. We correlated the different CT findings as lymph node size, shape, contour etc. with the histological findings in EBUS-TBNA performing t-tests and chi-square tests by means of a specialized computer algorithm (MedCalc $($ ) Software, Mariakerke, Belgium). Significance was set at a $P$ value of less than 0.05 . The study was approved by the ethics committee of our university and was HIPAA compliant. Patient informed consent was not felt necessary by the ethics committee.

\section{Results}

Of 59 retrospectively enrolled patients, cytological confirmed anthracosis (see Figure 3) was diagnosed in 53 lymph nodes in 39 patients. These patients (see Table 1) showed a mean age of 65.1 years (range 19-88 years) with a predominance of the male gender (28/39, 71.8\%). The control group of 20 patients showed a comparable age (mean 61.3 years, range 2996) but a more balanced sex ratio ( $55 \%$ vs. $45 \%)$; this difference was not significant $(p=0.44)$.

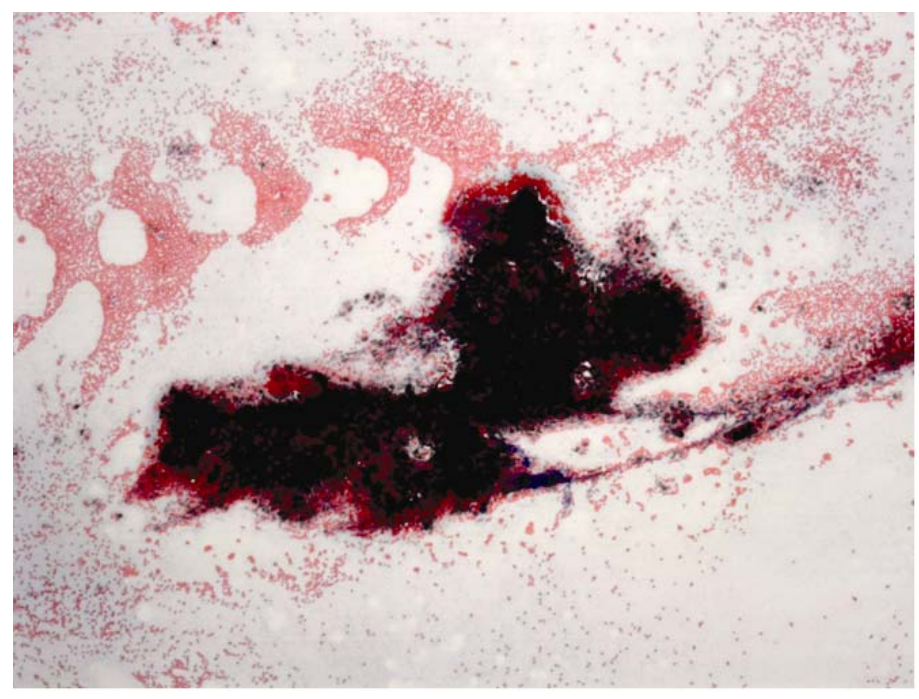

Figure 3. Cytologic specimen from an EBUS-TBNA with a fibrous fragment of a lymph node with deposition of anthracotic pigment in macrophages and in the extracellular space. At the periphery of the microphotograph, erythrocytes, some inflammatory cells and some normal epithelial cells are present. Papanicolaou staining, 5x.

Table 1. Distribution and demographic data of the 59 enrolled patients.

\begin{tabular}{lll}
\hline & anthraccosis & control group \\
\hline patients (n) & 39 & 20 \\
male (\%) & $72 \%$ & $55 \%$ \\
female (\%) & $28 \%$ & $45 \%$ \\
mean age (years) & 65,1 (range, 19-88) & 61,3 (range, 29-96) \\
EBUS-TBNA confirmed lymph nodes (n) & 53 & 27 \\
\hline
\end{tabular}

The retrospective analysis of the electronic health records of the 39 patients with lymph node enlargement due to anthracosis yielded that 7 patients had accompanying malignancy (5 cases of bronchial cancer, 2 cases of NHL). In 17 patients severe nicotine abuse was documented and only one patient had occupational exposure to dust. 
The control group (lymph node enlargement due to other benign conditions) comprised 20 patients with 27 examined enlarged lymph nodes. EBUS-TBNA revealed chronic lymphadenitis in 14 nodes. Pathologically this finding represents a nonspecific reactive hyperplasia of lymph nodes that is most commonly caused by an asymptomatic inflammatory process (pyogenic bacteria, mycobacteria, toxoplasmosis, HIV, infectious mononucleosis etc.) or by specific infectious agents that had disappeared before detection ${ }^{[15]}$. Of these patients only one showed pneumonia in CT. Sarcoidosis was diagnosed in 13 lymph nodes.

Comparing the distribution of EBUS confirmed enlarged lymph nodes on the ATS regions (see Table.2) we found that the most common site of benign enlarged lymph nodes was ATS region 7 (totally 32 nodes, i.e. 40\%). This holds for anthracotic lymph nodes as well as nodes of our control group. On closer inspection of the other regions (see Table.3) a difference was seen between the two groups regarding an accumulation of anthracotic lymph nodes on the right side (2R, $4 \mathrm{R}, 10 \mathrm{R}, 11 \mathrm{R})$. In these regions $25 / 31$ (81\%) of all enlarged nodes due to anthracotic were found. The distribution of enlarged lymph nodes due to sarcoidosis or lymphadenitis was more balanced. This difference was statistically significant $(p=0.04)$.

Table 2. Distribution of the EBUS confirmed anthracotic and other benign lymph nodes to the ATS regions. The most common site of benign enlarged lymph nodes was ATS region 7 (totally 32 nodes, i.e. 40\%).

\begin{tabular}{llll}
\hline ATS - region & anthracosis & other benign & Total (\%) \\
\hline $2 \mathrm{R}$ & 3 & 1 & $4(5)$ \\
$2 \mathrm{~L}$ & 1 & 0 & $1(1.25)$ \\
$4 \mathrm{R}$ & 4 & 4 & $8(10)$ \\
$4 \mathrm{~L}$ & 4 & 1 & $5(6.25)$ \\
7 & 22 & 10 & $32(40)$ \\
$10 \mathrm{R}$ & 9 & 3 & $12(15)$ \\
$10 \mathrm{~L}$ & 1 & 3 & $4(5)$ \\
$11 \mathrm{R}$ & 9 & 1 & $10(12.5)$ \\
$11 \mathrm{~L}$ & 0 & 4 & $4(5)$ \\
total & 53 & 27 & $80(100)$ \\
\hline
\end{tabular}

Table 3. Distribution of the EBUS confirmed anthracotic and other benign lymph nodes to the right and left side: On closer inspection of the other regions a significant difference was seen between the two groups regarding an accumulation of anthracotic lymph nodes on the right side $(p=0.04)$.

\begin{tabular}{llll}
\hline localisation & anthracosis (\%) & other (\%) & total (\%)) \\
\hline 2R, 4R, 10R, 11R & $25(80.6)$ & $9(52.9)$ & $34(70.8)$ \\
2L, 4L, 10L, 11L & $6(19.4)$ & $8(47.1)$ & $14(29.2)$ \\
total & $31(100)$ & $17(100)$ & $48(100)$ \\
\hline
\end{tabular}

EBUS (see Table 4) demonstrated significantly more often an indistinct margin in sarcoidosis and chronic lymphadenopathy than in enlarged lymph nodes due to anthracosis ( $25.9 \%$ vs. $5.7 \%, p=0.025)$. Anthracotic lymph nodes 
more often showed a heterogenous echogenicity than enlarged lymph nodes due to other benign conditions (28.3 vs. 11.1), but this difference was not significant $(p=0.15)$. CT findings (see Table 4)

Table 4. CT and EBUS findings in enlarged lymph nodes due to anthracosis or other benigne conditions (control group). The margins of anthracotic lymph nodes are significantly more often clear defined as well in CT as EBUS. Anthracotic lymph nodes demonstrate significantly more often hyperdensities like small calcifications.

\begin{tabular}{|c|c|c|c|c|c|c|}
\hline & & \multicolumn{2}{|c|}{ Anthracosis } & \multicolumn{2}{|c|}{ Control group } & \multirow{2}{*}{$\begin{array}{l}\text { Significance } \\
\text { n.s. } p=0.299\end{array}$} \\
\hline \multirow{4}{*}{ size } & Short axis & $13.7 n$ & & $14.5 n$ & & \\
\hline & Long axis & $19.3 n$ & & $20.4 n$ & & n.s. $p=0.359$ \\
\hline & & $\%$ & $\mathrm{n}$ & $\%$ & $\mathrm{n}$ & \\
\hline & \multicolumn{5}{|c|}{ CT findings } & \\
\hline \multirow{3}{*}{ shape } & Oval & 84.9 & 45 & 85.2 & 23 & n.s. $p=0.91$ \\
\hline & Round & 15.1 & 5 & 11.1 & 3 & \multirow{5}{*}{ sign. $p=0.023$} \\
\hline & polycyclic & 5.7 & 3 & 3.7 & 1 & \\
\hline \multirow{3}{*}{ margin } & ill defined & 1.9 & 1 & 18.5 & 5 & \\
\hline & clear defined & 66 & 35 & 48.1 & 13 & \\
\hline & confluence & 32.1 & 17 & 33.3 & 9 & \\
\hline Contrast enhancement & & 3.8 & 2 & 3.7 & 1 & n.s. $p=0.99$ \\
\hline calcification & & 24.5 & 13 & 3.7 & 1 & sign. $p=0.017$ \\
\hline fat & & 3.8 & 2 & 3.7 & 1 & n.s. $p=0.99$ \\
\hline colliquation & & 0 & 0 & 0 & 0 & - \\
\hline \multicolumn{7}{|c|}{ EBUS Findings } \\
\hline \multirow{2}{*}{ margin } & indistinct & 5.7 & 3 & 25.9 & 7 & \multirow[t]{2}{*}{ sign. $p=0.025$} \\
\hline & distinct & 94.3 & 50 & 74.1 & 20 & \\
\hline \multirow{2}{*}{ echogenity } & homogenous & 71.7 & 38 & 88.9 & 24 & \multirow[t]{2}{*}{ n.s. $p=0.154$} \\
\hline & heterogenous & 28.3 & 15 & 11.1 & 3 & \\
\hline total & & 100 & 53 & 100 & 27 & \\
\hline
\end{tabular}

The short axis of the histological proven enlarged anthracotic lymph nodes ranged from 8mm-23mm with a mean diameter of $13.7 \mathrm{~mm}$. The long axis ranged from $12 \mathrm{~mm}-31 \mathrm{~mm}$ with a mean diameter of $19.3 \mathrm{~mm}$. Enlarged lymph nodes in other benign conditions showed a mean short axis diameter of 14.5mm (range: $8 \mathrm{~mm}-27 \mathrm{~mm}$ ) and a mean long axis diameter of 20.4mm (range: $10 \mathrm{~mm}-34 \mathrm{~mm})$. No significant differences were found regarding short and long axis diameters $(p>0.05$, respectively).

The majority of lymph nodes with signs of anthracosis showed an oval shape (45/53, i.e. $84.9 \%)$. Only five nodes were round- shaped (15.1\%), three nodes showed a polycyclic shape (5.7\%). In the control group, a similar frequency of the different types of shape was found: oval 23/27 (85.2\%), round shaped 3/24 (11.1\%), polycyclic 1/27 (3.7\%). Differences regarding nodal shape were not statistically significant.

Confluence of two or more enlarged lymph nodes (see Figure 4) was seen in 17/53 (32.1\%) in anthracosis and in 9/27 (33.3\%) of the control group $(p>0.05)$. Contrast enhancement was documented in 2/53 (3.8\%) of the anthracotic lymph 
nodes and in $1 / 27$ (3.7\%) of lymph nodes in the control group $(p>0.05)$. Central hypodensity due to fatty involution was seen in two of the anthracotic lymph nodes (3.8\%), and in one patient (3.7\%) of the control group $(p>0.05)$. Lymph node necrosis was neither seen in lymph node anthracosis nor in nodes of the control group.

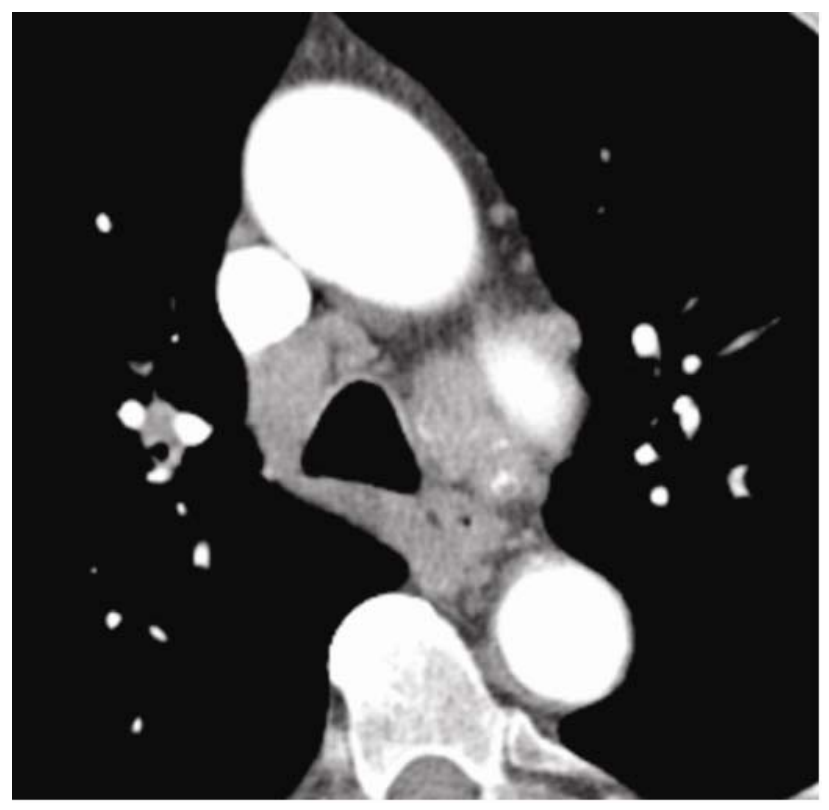

Figure 4. 81 year old male, bronchial cancer in the right lower lobe. Chest CT (MSCT $64 \mathrm{~mm} \times 0.75 \mathrm{~mm}$ slice collimation, tube voltage $120 \mathrm{kV}$ ) shows enlarged paratracheal lymph nodes in the ATS region 4R and 4L; subtle hyperdensities like calcifications, moderate polycyclic shape, nodal confluence. Cytological examination revealed deposition of anthracotic pigment without any evidence for malignancy.

In summary, only two significant differences between the two groups were found: anthracotic lymph nodes more often showed calcifications (see Figure 4). This finding was demonstrated in 13/53 (24.5\%) of anthracotic nodes and in only one node (3.7\%) of the control group ( $p=0.017)$. While the vast majority of enlarged lymph nodes in anthracosis were clearly defined (98.1\%), 5/27 (18.5\%) of benign enlarged lymph nodes in the control group were accurately ill-defined from the surrounding tissue $(p=0.01)$. In the latter, EBUS-TBNA revealed three cases of sarcoidosis and two cases of lymphadenitis. Consentaneously, EBUS had shown a significant higher percentage of lymph nodes with indistinct margins in these patients $(25.9 \%$ vs. $5.7 \%, p=0.025)$.

\section{Discussion}

Anthracosis is a form of pneumoconiosis which is not only caused by coal dust but also other environmental factors such as air pollution, biomass fuels used extensively for cooking ("hut lung”), and cigarette smoking ${ }^{[9,16-19]}$. A recently published comparative study on 44 heavy smokers and 44 non- smokers demonstrated that enlarged mediastinal lymph nodes may occur in up to $52 \%$ of heavy smokers, especially in those with severe bronchitis ${ }^{[9]}$. The authors of that study assumed that lymph node enlargement in smokers may be caused by a deposition of anthracotic material and dust induced response. One aim of the present comparative study between CT and EBUS-TBNA was to examine the validity of this hypothesis.

The findings of the present study confirm our hypothesis that anthracosis may result in a considerable enlargement of mediastinal lymph nodes up to $23 \mathrm{~mm}$ in short axis diameter. 
The comparison of enlarged lymph nodes caused by lymphadenopathy, sarcoidosis and anthracosis showed some different findings.

First, lymph node calcification was significantly more often observed in anthracotic nodes than in lymph node enlargement due to other benign conditions. The finding that lymph node calcifications were observed in approximately one third of the anthracotic lymph nodes is in accordance to the literature ${ }^{[20-22]}$.

Second, we significantly more often found ill-defined margins in sarcoidosis and lymphadenitis than in anthracosis. This holds for CT as well as the ultrasound examination. Accordingly, Sakai et al found that the ill-defined margin of an enlarged lymph node is a relatively common feature in inflammatory changed lymph nodes ${ }^{[23]}$.

However, all other examined CT features showed very similar findings in the two groups.

As a consequence, no significant differences were observed regarding the size, shape (most often oval), presence of contrast enhancement or fatty involution.

While some authors assessed nodal confluence as a reliable indicator of malignancy ${ }^{[24]}$, surprisingly this finding was observed as well in nearly one third of the anthracotic as benign enlarged lymph nodes in our study. Therefore we think that confluence of enlarged lymph nodes cannot be interpreted as a sign of metastatic disease. On the other hand lymph node necrosis, that has not only been reported for malignancy, but also in nodal inflammation, was not seen in any of the benign enlarged nodes of our study. As a consequence, nodal colliquation might be interpreted as a strong indicator for nodal malignancy. However, for a confirmation of this fact, an examination of a greater cohort of patients would be necessary.

Today, the still existing need for surgical staging of lung cancer often seems to be underestimated ${ }^{[25]}$ and chest CT remains the standard imaging technique for the preoperative staging ${ }^{[26]}$. In contrast, a recently published study based on histological confirmed lymph nodes by means of EBUS-TBNA revealed a low sensitivity of $77 \%$ and a specificity of $55 \%$ for the CT based diagnosis of lymph node involvement in lung cancer ${ }^{[27]}$. The main cause for the insufficient specificity of CT seems to be that mediastinal lymph node enlargement occurs in a lot of benign conditions ${ }^{[3]}$. The findings of our study suggest that especially anthracotic lymph nodes may mimic mediastinal lymph node metastases, due to the considerable sizes and nodal confluence. This knowledge may avoid overstaging of malignant disease.

Although FDG-PET has now become a standard diagnostic procedure, recent studies revealed that the diagnostic accuracy

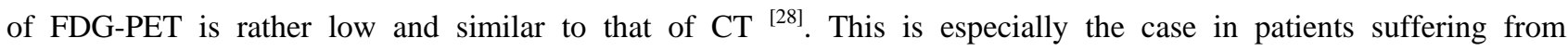
pneumoconiosis ${ }^{[29-31]}$. Consequently, histological confirmation of abnormal lymph nodes on PET/CT is important in many cases, too. In accordance, Fielding et Kurimoto recently postulated that EBUS-TBNA staging is needed for any patient with CT lymph nodes greater than $1 \mathrm{~cm}$ in short axis, or PET-positive mediastinal nodes ${ }^{\text {[32] }}$. But until yet, the impact of EBUS-TBNA on the use of diagnostic modalities for tissue acquisition in patients with lung cancer is not definitely known ${ }^{[32]}$. Nevertheless, Jose et al already showed in a recently published study on 316 patients that the increasing use of EBUS-TBNA has led to a significant reduction in standard bronchoscopy and mediastinoscopy ${ }^{[33]}$. Furthermore, Dong et al showed in a meta-analysis comprising 9 studies with a total of 1066 patients that EBUS-TBNA has an excellent sensitivity, specificity and accuracy in the mediastinal staging of NSCLC ${ }^{[34]}$. But this holds not only for the diagnosis of metastatic disease: Cordovilla et al showed in a recently published study on 49 cases in which diagnosis was performed by EBUS-TBNA that the sensitivity of EBUS-TBNA was 74\% and the diagnostic accuracy was $79 \%$ in lymph node enlargement due to sarcoidosis ${ }^{[35]}$. The authors concluded that EBUS-TBNA is not only a useful tool but should be nowadays the first step in the diagnosis of sarcoidosis if enlarged hilar lymph nodes are present in CT. This opinion is supported by Hong et al ${ }^{[36]}$; these authors performed a comparative study in 33 patients which underwent EBUS-TBNA, TBLB and BAL during the same session. In this setting EBUS-TBNA was the most sensitive method for diagnosing stage I and II sarcoidosis. 


\section{Limitations}

There were some limitations to our study. First, the sample size was small, preventing a generalization of our results. Second, the study design was retrospective and therefore some clinical data may be questioned (e.g. percentage of smokers). Another, but methodological based limitation seems to be that not all enlarged nodes present on CT could have been evaluated.

In the present study no comparison has been presented with special regard to non-benign causes of lymphadenopathy. Therefore we only can infer that anthracosis could mimic malignancy from the fact that some of the anthracotic lymph nodes were enlarged on CT. Therefore we consider our results preliminary.

\section{Conclusion}

Anthracosis may result in an enlargement of mediastinal lymph nodes, showing often confluence and calcifications on CT. Although there are some different findings (anthracotic nodes show more often show calcifications, but less often are ill-defined) these lymph nodes cannot definitely be differentiated from other benign enlarged nodes caused by chronic lymphadenitis or sarcoidosis.

\section{References}

[1] Hunt BM, Vallières E, Buduhan G, Aye R, Louie B. Sarcoidosis as a benign cause of lymphadenopathy in cancer patients. Am J Surg. 2009; 197: 629-32. http://dx.doi.org/10.1016/j.amjsurg.2009.01.004

[2] Ardekani MS, Issa M, Green L. Diagnostic and economic impact of heart failure induced mediastinal lymphadenopathy. Int J Cardiol. 2006; 109: 137-8. http://dx.doi.org/10.1016/j.ijcard.2005.04.011

[3] Kirchner J, Kirchner EM, Goltz JP, Obermann A, Kickuth R. Enlarged hilar and mediastinal lymph nodes in chronic obstructive pulmonary disease. J Med Imaging Radiat Oncol. 2010; 54: 333-8. http://dx.doi.org/10.1111/j.1754-9485.2010.02179.x

[4] Baldwin DR, Lambert L, Pantin CF, Prowse K, Cole RB. Silicosis presenting as bilateral hilar lymphadenopathy. Thorax. 1996; 51: 1165- 7. http://dx.doi.org/10.1136/thx.51.11.1165

[5] Hartung W, Moon JS. Current pattern of anthracosilicosis, its complications and correlation with other diseases (evaluation of 300 legal autopsies 1977-1988). Pneumologie. 1992, 46: 516-24.

[6] McLoud TC. Occupational lung disease. Radiol Clin North Am. 1991; 29: 931-41.

[7] Varela-Lema L, Fernández-Villar A, Ruano-Ravina A. Effectiveness and safety of endobronchial ultrasound-transbronchial needle aspiration: a systematic review. Eur Respir J. 2009; 33: 1156-64. http://dx.doi.org/10.1183/09031936.00097908

[8] Ooi CG, Khong PL, Cheng RS, Tan B, Tsang F, Lee I, Lam VS, Leung KK, Tsang KW. The relationship between mediastinal lymph node attenuation with parenchymal lung parameters in silicosis. Int J Tuberc Lung Dis. 2003; 7: 1199-206.

[9] Kirchner J, Kirchner EM, Goltz JP, Lorenz VW, Kickuth R. Prevalence of enlarged mediastinal lymph nodes in heavy smokers-a comparative study Eur Radiol. 2011; 21: 1594-9. http://dx.doi.org/10.1007/s00330-011-2111-9

[10] Glazer GM, Gross BH, Quint LE, Francis IR, Bookstein FL, Orringer MB. Normal mediastinal lymph nodes: number and size according to American Thoracic Society mapping. AJR Am J Roentgenol. 1985; 144: 261- 5. http://dx.doi.org/10.2214/ajr.144.2.261

[11] Pombo F, Rodríguez E, Mato J, Pérez-Fontán J, Rivera E, Valvuena L. Patterns of contrast enhancement of tuberculous lymph nodes demonstrated by computed tomography. Clin Radiol. 1992; 46: 13- 7. http://dx.doi.org/10.1016/S0009-9260(05)80026-1

[12] American Thoracic Society. Medical section of the American Lung Association. Clinical staging of primary lung cancer. Am Rev Respir Dis. 1983; 127: 659- 64.

[13] Herth FJ, Ernst A, Eberhardt R, Vilmann P, Dienemann H, Krasnik M. Endobronchial ultrasound-guided transbronchial needle aspiration of lymph nodes in the radiologically normal mediastinum. Eur Respir J. 2006; 28: 910-4. http://dx.doi.org/10.1183/09031936.06.00124905

[14] Fujiwara T, Yasufuku K, Nakajima T, Chiyo M, Yoshida S, Suzuki M, et al. The utility of sonographic features during endobronchial ultrasound-guided transbronchial needle aspiration for lymph node staging in patients with lung cancer: a standard endobronchial ultrasound image classification system. Chest. 2010; 138: 641-7. http://dx.doi.org/10.1378/chest.09-2006 
[15] Natella V, Cozzolino I, Sosa Fernandez LV, Vigliar E. Lymph nodes fine needle cytology in the diagnosis of infectious diseases: clinical settings. Infez Med. 2012; 20 Suppl 3: 12- 5.

[16] Gold JA, Jagirdar J, Hay JG, Addrizzo-Harris DJ, Naidich DP, Rom WN. Hut lung. A domestically acquired particulate lung disease. Medicine (Baltimore). 2000; 79: 310-7. http://dx.doi.org/10.1097/00005792-200009000-00004

[17] Kim YJ, Jung CY, Shin HW, Lee BK. Biomass smoke induced bronchial anthracofibrosis: presenting features and clinical course. Respir Med. 2009; 103: 757-65. http://dx.doi.org/10.1016/j.rmed.2008.11.011

[18] Klaaver M, Kars AH, Maat AP, den Bakker MA. Pseudomediastinal fibrosis caused by massive lymphadenopathy in domestically acquired particulate lung disease. Ann Diagn Pathol. 2008; 12: 118-21. http://dx.doi.org/10.1016/j.anndiagpath.2007.07.004

[19] Naccache JM, Monnet I, Nunes H, Billon-Galland MA, Pairon JC, Guillon F, et al. Anthracofibrosis attributed to mixed mineral dust exposure: report of three cases. Thorax. 2008; 63: 655-7. http://dx.doi.org/10.1136/thx.2006.070243

[20] Bilici A, Erdem T, Boysan SN, Acbay O, Oz B, Besirli K, et al. A case of anthracosis presenting with mediastinal lymph nodes mimicking tuberculous lymphadenitis or malignancy. Eur J Intern Med. 2003; 14: 444- 446. http://dx.doi.org/10.1016/j.ejim.2003.09.001

[21] Hartung W, Moon JS.[Current pattern of anthracosilicosis, its complications and correlation with other diseases (evaluation of 300 legal autopsies 1977-1988). Pneumologie. 1992; 46: 516-24.

[22] Vahlensieck M, Overlack A, Müller KM.Computed tomographic high-attenuation mediastinal lymph nodes after aluminum exposition. Eur Radiol. 2000; 10: 1945-6. http://dx.doi.org/10.1007/s003300000534

[23] Sakai O, Curtin HD, Romo LV, Som PM. Lymph node pathology. Benign proliferative, lymphoma, and metastatic disease. Radiol Clin North Am. 2000; 38: 979-98. http://dx.doi.org/10.1016/S0033-8389(05)70216-5

[24] Stern WB, Silver CE, Zeifer BA, Persky MS, Heller KS. Computed tomography of the clinically negative neck. Head Neck. 1990; 12: 109-13. http://dx.doi.org/10.1002/hed.2880120203

[25] Vansteenkiste J, Dooms C, De Leyn P. Early stage non-small-cell lung cancer: challenges in staging and adjuvant treatment: evidence-based staging. Ann Oncol. 2010; 21: 189-95. http://dx.doi.org/10.1093/annonc/mdq424

[26] Paul NS, Ley S, Metser U. Optimal imaging protocols for lung cancer staging: CT, PET, MR imaging, and the role of imaging. Radiol Clin North Am. 2012; 50: 935-49. http://dx.doi.org/10.1016/j.rcl.2012.06.007

[27] Yasufuku K, Nakajima T, Motoori K, Sekine Y, Shibuya K, Hiroshima K, Fujisawa T.Comparison of endobronchial ultrasound, positron emission tomography, and CT for lymph node staging of lung cancer. Chest. 2006; 130: 710-8. http://dx.doi.org/10.1378/chest.130.3.710

[28] Lin WY, Hsu WH, Lin KH, Wang SJ. Role of preoperative PET-CT in assessing mediastinal and hilar lymph node status in early stage lung cancer. J Chin Med Assoc. 2012; 75: 203-8. http://dx.doi.org/10.1016/j.jcma.2012.04.004

[29] Cheng NM, Yeh TW, Ho KC, Ng SH, Hsueh C, Yen TC, Liao. CT False positive F-18 FDG PET/CT in neck and mediastinum lymph nodes due to anthracosis in a buccal cancer patient. Clin Nucl Med. 2011; 36: 963-4.

http://dx.doi.org/10.1097/RLU.0b013e3182291f11

[30] Reichert M, Bensadoun ES PET imaging in patients with coal workers pneumoconiosis and suspected malignancy. J Thorac Oncol. 2009; 4: 649-51. http://dx.doi.org/10.1097/JTO.0b013e31819d4778

[31] Saydam O, Gokce M, Kilicgun A, Tanriverdi O. Accuracy of positron emission tomography in mediastinal node assessment in coal workers with lung cancer. Med Oncol. 2012; 29: 589-94. http://dx.doi.org/10.1007/s12032-011-9879-y

[32] Fielding D, Kurimoto N. EBUS-TBNA staging of lung cancer. Clin Chest Med. 2013; 34: 385-94. http://dx.doi.org/10.1016/j.ccm.2013.06.003

[33] José RJ1, Shaw P, Taylor M, Lawrence DR, George PJ, Janes SM, et al. Impact of EBUS-TBNA on modalities for tissue acquisition in patients with lung cancer. QJM. 2014; 107: 201-6. http://dx.doi.org/10.1093/qjmed/hct233

[34] Dong X1, Qiu X, Liu Q, Jia J. Endobronchial ultrasound-guided transbronchial needle aspiration in the mediastinal staging of non-small cell lung cancer: a meta-analysis. Ann Thorac Surg. 2013; 96: 1502-7. http://dx.doi.org/10.1016/j.athoracsur.2013.05.016

[35] Cordovilla R, Torracchi A, Varela Simó G, Gomez MA, González-Ruiz JM, Jiménez López MF, Lanchas M, Barrueco M. The Role of EBUS-TBNA for the Diagnosis of Sarcoidosis. Chest. 2014; 145:498A. http://dx.doi.org/10.1378/chest.1824354

[36] Hong G1, Lee KJ, Jeon K, Koh WJ, Suh GY, Chung MP, et al. Usefulness of endobronchial ultrasound-guided transbronchial needle aspiration for diagnosis of sarcoidosis. Yonsei Med J. 2013; 54: 1416-21. http://dx.doi.org/10.3349/ymj.2013.54.6.1416 